\title{
Bin1: A New Player in IBD Barrier Dysfunction
}

\author{
Hyunji Ryu - Daniela Posca $\cdot$ Terrence Barrett
}

Received: 23 April 2012/Accepted: 30 April 2012/Published online: 30 May 2012

(C) Springer Science+Business Media, LLC 2012

The ability of the host to regulate permeability of the intestinal barrier is a critical determinant of host defense. Regulation of permeability occurs at the level of epithelial tight junctions (TJ). TJ are composed of transmembrane proteins, such as occludin, claudin, and JAM family proteins. Plaque proteins, such as zonula occludins ( $\mathrm{ZO}$ ), connect the TJ complex to F-actin and actomyosin rings regulating cytoskeletal reorganization (Fig. 1, reviewed in [1]). Several signaling pathways, such as myosin light chain kinase (MLCK), protein kinase C (PKC), mitogenactivated protein kinases (MAPK), and the Rho family of small GTPases, control the remodeling and maintenance of TJ. TJ not only regulate paracellular transport of nutrients and water, but they also provide a barrier against enteric microbes. Although the exact etiology is unknown, there is ample evidence that epithelial barrier function is compromised in inflammatory bowel disease (IBD) patients. Data from various studies using inert non-metabolized probes indicate that paracellular permeability is increased in Crohn's Disease (CD) [2]. Furthermore, increased permeability may precede the onset of inflammation [3]. Researchers also found that enteric permeability is increased in 10-54\% of healthy, first-degree relatives of IBD patients $[4,5]$. To date, several studies have associated IBD with mutations in the NOD2 gene [6, 7]. However,

\footnotetext{
H. Ryu $\cdot$ D. Posca $\cdot$ T. Barrett $(\bowtie)$

Department of Internal Medicine, Division of Gastroenterology, Northwestern University Feinberg School of Medicine, 300 E. Chicago Ave., Tarry 4-725, Chicago, IL 60611, USA e-mail: tabarrett@ northwestern.edu

H. Ryu

e-mail: h-ryu@northwestern.edu

D. Posca

e-mail: d-posca@northwestern.edu
}

NOD2 penetrance of the most at-risk genotypes is low [8], and it is likely that cooperation with other genetic factors, such as TJ defects, is required for disease development. Interestingly, Buhner et al. detected increased permeability in IBD patients and family members with NOD2 mutations [9]. Altogether, these findings support the speculation that altered permeability constitutes one of several genetically determined variables that enhances the risk for developing IBD.

The notion that altered epithelial permeability predisposes patients to IBD is supported by data from several animal models with transmucosal barrier defects. Using transgenic mice that express constitutively active MLCK, $\mathrm{Su}$ et al. demonstrated that, while insufficient to fully induce experimental colitis alone, TJ defects exacerbated disease in the adoptive transfer colitis model [10]. Other models of barrier disruption, such as dominant negative $\mathrm{N}$-cadherin expression, resulted in spontaneous inflammation [11], further enforcing the link between barrier dysfunction and immune homeostasis. Altered permeability may also occur due to effects of local cytokines. Tumor necrosis factor, a cytokine critical for the pathogenesis of IBD, activates MLCK, resulting in TJ disruption via internalization of occludin from the cell membrane [12]. Also, interleukin-13, a cytokine upregulated in ulcerative colitis, induces expression of the pore-forming TJ protein claudin-2, leading to barrier dysfunction [13]. Altogether, these data represent the dynamic relationship between inflammation and permeability and how their imbalance may result in the development of IBD.

In this issue of Digestive Diseases and Sciences, Chang et al. present data that further support the tie between TJ permeability and IBD pathogenesis via the protein Bridging integrator 1 (Bin1) [14]. Binl is a member of the Bin amphiphysin rys (BAR) adaptor family with myriad 
biological functions, including cytoskeletal organization, tumor suppression, transcriptional regulation, and DNA repair $[15,16]$. Binl homologs are found in yeast and Caenorhabditis elegans, supporting the argument for evolutionary conservation of function (reviewed in [17]). Chang et al. also describe a novel function of Binl in regulating TJ permeability during experimental colitis. The authors previously generated Binl mosaic knockout (KO) mice in which genetic deletions occur throughout the tissue with intermixing of cells harboring recombined $\left(\mathrm{Binl}^{-{ }^{-}}\right)$ or wild-type $\left(\right.$ Binl $\left.^{+/+}\right)$alleles. Binl attenuation had no effect on baseline histology, but it did enhance resistance to dextran sodium sulfate (DSS)-induced colitis. Binl attenuation increased survival and tempered disease activity, as manifested by decreases in diarrhea, bloody stools, and weight loss. Decreased Binl also enhanced epithelial barrier function, measured by increased basal transepithelial electrical resistance (TER) and decreased paracellular mannitol flux. Importantly, the resistant phenotype was specific for inflammation-induced stimuli, as no difference was observed in chemically induced permeability by phorbol ester (PBDU) or sodium caprate. Chang et al. suggest that enhanced barrier function mitigated epithelial damage and inflammatory cell infiltration, possibly by preventing bacterial translocation and/or diffusion of proinflammatory cytokines. Thus, these findings suggest that Binl enhances permeability shifts in the setting of inflammation.

Thus far, most IBD therapies heavily rely on immunosuppressive drugs, including corticosteroids and antibodies against pro-inflammatory cytokines. Despite advances in immunosuppressive therapy, IBD remains a challenging disease to treat. The current study by Chang et al. proposes epithelial Binl as a novel therapeutic target without relying on immunosuppression. The data presented suggest that inhibition of Binl could enhance intestinal barrier function and thereby attenuate disease. Another potential benefit of Binl blockade is enhanced epithelial regeneration after inflammation-induced damage (Fig. 1). Within the mammalian system, Binl interacts with c-myc to restrict oncogenic and transcriptional properties [15]. Reductions in Binl expression are observed at high frequencies in lung, prostate, and metastatic cancer [18]. In the intestine, Bin 1 deletion exacerbates Ras-mediated carcinogenesis, and attenuated expression correlates with tumor progression $[18,19]$. Although it is unclear whether Binl is involved in c-myc regulation in colitis, Binl inhibition may enhance c-myc functions. Cellular proliferation is a key step in mucosal regeneration (reviewed in [20]). As c-myc participates in cellular proliferation, Binl inhibition may further benefit IBD patients by promoting wound healing. This is especially important because clinicians consider mucosal healing to be a reliable indicator of clinical

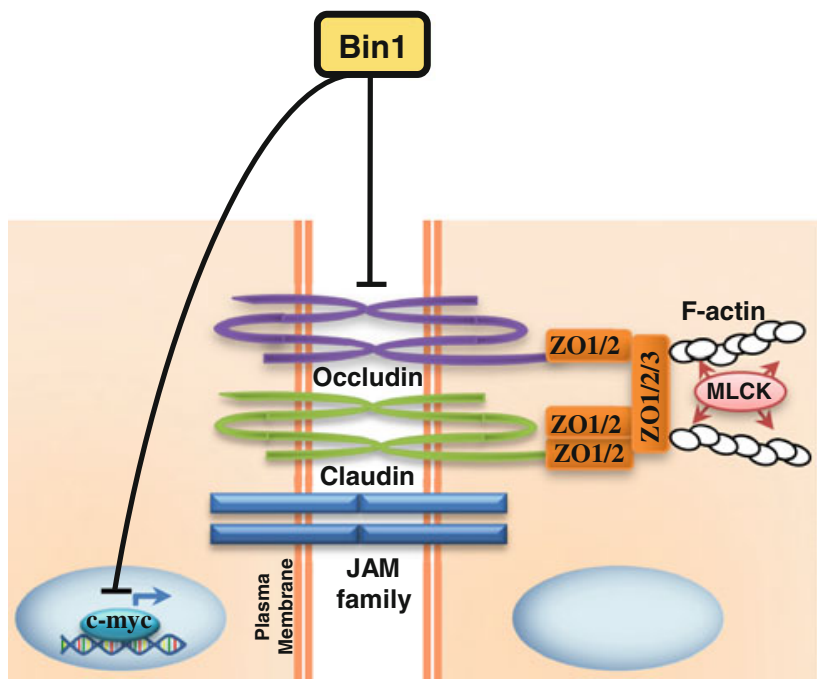

Fig. 1 Bridging integrator (Bin1) positively regulates intestinal permeability: potential sites of action. Shown are sites where Binl may affect mucosal permeability. Bin1 may interact directly with epithelial tight junction (TJ) proteins and/or occludin endocytic recycling. It is also possible that Binl impacts c-myc transcriptional activities that affect proteins involved in proliferation and permeability. $M L C K$ myosin light chain kinase, $Z O$ zonula occludins. Figure is adapted from Ulluwishewa et al. [1]

efficacy of therapeutic agents [21]. Despite the therapeutic potential of Binl inhibition, however, one must consider the potential risk for dysplasia development, as Binl is an intestinal tumor suppressor and colitis increases the risk for colorectal cancer.

It remains to be determined precisely how Bin1 participates in the regulation of barrier function during colitis. Immunohistochemical analysis revealed cytosolic and membrane-associated staining patterns of Binl in colonic epithelial cells [18]. One speculation is that Binl regulates endocytic recycling and subcellular rearrangement of TJ proteins, such as occludin, which is a critical step in TJ regulation. While the authors found no significant change in occludin expression upon Binl attenuation, it is plausible that subcellular localization of occludin and other members of the TJ complex were altered. Several studies have linked C. elegans, mammalian Binl [22], and other members of the BAR protein family to endocytosis and endocytic recycling. Endocytosis of occludin is a critical step in TJ remodeling, as demonstrated by the attenuation of cytokine-induced intestinal permeability following pharmacological inhibition of endocytosis [12]. Thus, the effects of Binl on mucosal permeability may be due, in part, to alterations in occludin endocytic recycling (Fig. 1).

IBD has long been considered to result from the combination of immune dysregulation and altered mucosal permeability. Data presented by Chang et al. further support the hypothesis that compromised permeability predisposes the host to IBD. The authors present a novel 
animal model that points to targeting Binl as a means of strengthening mucosal barrier function in the colon. As combination therapies become increasingly common, it is attractive to speculate that the topical administration of Binl small molecule inhibitors may serve as a useful adjunct to systemic immunomodulatory agents.

\section{References}

1. Ulluwishewa D, Anderson RC, McNabb WC, Moughan PJ, Wells JM, Roy NC. Regulation of tight junction permeability by intestinal bacteria and dietary components. J Nutr. 2011;141: 769-776.

2. Hollander D, Vadheim CM, Brettholz E, Petersen GM, Delahunty $\mathrm{T}$, Rotter JI. Increased intestinal permeability in patients with Crohn's disease and their relatives. A possible etiologic factor. Anna Internal Med. 1986;105:883-885.

3. Madsen KL, Malfair D, Gray D, Doyle JS, Jewell LD, Fedorak RN. Interleukin-10 gene-deficient mice develop a primary intestinal permeability defect in response to enteric microflora. Inflamm Bowel Dis. 1999;5:262-270.

4. Hollander D. Permeability in Crohn's disease: altered barrier functions in healthy relatives? Gastroenterology. 1993;104:18481851.

5. May GR, Sutherland LR, Meddings JB. Is small intestinal permeability really increased in relatives of patients with Crohn's disease? Gastroenterology. 1993;104:1627-1632.

6. Ogura Y, Bonen DK, Inohara N, et al. A frameshift mutation in NOD2 associated with susceptibility to Crohn's disease. Nature. 2001;411:603-606.

7. Hugot JP, Chamaillard M, Zouali H, et al. Association of NOD2 leucine-rich repeat variants with susceptibility to Crohn's disease. Nature. 2001;411:599-603.

8. Hugot JP, Zaccaria I, Cavanaugh J, et al. Prevalence of CARD15/ NOD2 mutations in Caucasian healthy people. Am J Gastroenterol. 2007;102:1259-1267.

9. Buhner S, Buning C, Genschel Jet al. Genetic basis for increased intestinal permeability in families with Crohn's disease: role of CARD15 3020insC mutation? Gut. 2006;55:342-347.
10. Su L, Shen L, Clayburgh DR, et al. Targeted epithelial tight junction dysfunction causes immune activation and contributes to development of experimental colitis. Gastroenterology. 2009; 136:551-563.

11. Hermiston ML, Gordon JI. Inflammatory bowel disease and adenomas in mice expressing a dominant negative $\mathrm{N}$-cadherin. Science. 1995;270:1203-1207.

12. Marchiando AM, Shen L, Graham WV, et al. Caveolin-1-dependent occludin endocytosis is required for TNF-induced tight junction regulation in vivo. J Cell Biol. 2010;189:111-126.

13. Heller F, Florian P, Bojarski C, et al. Interleukin-13 is the key effector Th2 cytokine in ulcerative colitis that affects epithelial tight junctions, apoptosis, and cell restitution. Gastroenterology. 2005; 129:550-564.

14. Chang MY, Boulden J, Valenzano MC, et al. Bin1 attenuation suppresses experimental colitis by enforcing intestinal barrier function. Dig Dis Sci. (Epub ahead of print). doi:10.1007/ s10620-012-2147-y.

15. Sakamuro D, Elliott KJ, Wechsler-Reya R, Prendergast GC. BIN1 is a novel MYC-interacting protein with features of a tumour suppressor. Nat Genet. 1996;14:69-77.

16. Pineda-Lucena A, Ho CS, Mao DY, et al. A structure-based model of the $\mathrm{c}-\mathrm{Myc} / \mathrm{Bin} 1$ protein interaction shows alternative splicing of Bin1 and c-Myc phosphorylation are key binding determinants. J Mol Biol. 2005;351:182-194.

17. Cowling BS, Toussaint A, Muller J, Laporte J. Defective membrane remodeling in neuromuscular diseases: insights from animal models. PLoS Genet. 2012;8:e1002595.

18. DuHadaway JB, Lynch FJ, Brisbay S, et al. Immunohistochemical analysis of Bin1/Amphiphysin II in human tissues: diverse sites of nuclear expression and losses in prostate cancer. $J$ Cell Biochem. 2003;88:635-642.

19. Chang MY, Boulden J, Katz JB, et al. Bin1 ablation increases susceptibility to cancer during aging, particularly lung cancer. Cancer Res. 2007;67:7605-7612.

20. Okamoto R, Watanabe M. Cellular and molecular mechanisms of the epithelial repair in IBD. Digest Dis Sci. 2005;50:S34-S38.

21. Dave M, Loftus EV Jr. Mucosal healing in inflammatory bowel disease-a true paradigm of success? Gastroenterol Hepatol. 2012;8:29-38.

22. Pant S, Sharma M, Patel K, Caplan S, Carr CM, Grant BD. AMPH-1/Amphiphysin/Bin1 functions with RME-1/Ehd1 in endocytic recycling. Nat Cell Biol. 2009;11:1399-1410. 